\title{
Characterisation Studies of Silicon Photomultipliers for a Calorimeter for the ILC
}

\author{
Patrick Eckert, Wei Shen, Hans-Christian Schultz-Coulon, Rainer Stamen, \\ Alexander Tadday* \\ Kirchhoff Institute for Physics, University of Heidelberg \\ E-mail: ataddayakip.uni-heidelberg.de
}

\section{Kolja Prothmann, Frank Simon}

MPI for Physics and Excellence Cluster Universe, Munich

\section{Christian Kiesling, Olaf Reimann}

MPI for Physics, Munich

\author{
Erika Garutti, Martin Göttlich, Felix Sefkow \\ DESY, Hamburg
}

\begin{abstract}
The analogue hadronic calorimeter for the International Linear Collider, developed by the CALICE Collaboration, uses novel silicon pixel-detectors (Silicon Photomultipliers) for the readout of small organic scintillator tiles. In order to find the devices best suited for this application, several test-setups have been developed which allow to measure and compare the properties of such sensors. In the work presented here, we focus on an experimental method to determine the Photon-Detection-Efficiency over a wide spectral range. In addition, two test-setups are discussed which allow to systematically study the overall device uniformity by illumination of small areas of the sensors, one using a pulsed light-source and the other a continuous-wave laser beam. First results of such uniformity studies for different sensor-models are also presented.
\end{abstract}

International Workshop on New Photon Detectors PD09

June 24-26 2009

Shinshu University Matsumoto Japan

\footnotetext{
* Speaker.
} 


\section{Introduction}

Within the CALICE ${ }^{1}$ collaboration several calorimeter concepts for the future International Linear Collider (ILC) are studied. A concept of a highly granular analogue hadronic calorimeter (AHCAL), uses novel silicon photomultipliers for the readout of small organic scintillating tiles $[1,2]$. Since the attached SiPM (MEPHI/PULSAR) has its highest Photon-Detection-Efficiency (PDE) in the green spectral region, in the current physics prototype, a wavelength shifting fibre (WLSF) has been inserted on each tile to convert the mainly blue scintillation light into green light in order to increase the observable number of photons. In addition, the uniformity in response to particles traversing the tile at different positions is improved by the fibre. For future prototypes there are also different readout schemes under consideration, e.g. a direct readout without wavelength shifting fibre which may reduce the complexity of assembly. In this case a sensor with a high PDE in the blue spectral region would be needed to reach a sufficiently high detection efficiency for single minimum ionizing particles crossing the scintillator cell.

In order to classify the different sensor types available, a test-setup has been developed for measuring the SiPM PDEs over a wide spectral range. Further test-setups allow to study the uniformity of the SiPM response to a focussed light spot as well as position dependent measurements of the cross-talk probability. Results of such uniformity measurements, carried out at Heidelberg University and the Max-Planck Institut für Physik in Munich are presented and discussed in detail.

\section{PDE Measurement}

\subsection{Statistical Analysis}

In order to determine the PDE, the response of the device under test (SiPM) to a referenced light source has to be measured. A setup (see figure 1) has been developed which utilises an integrating sphere ${ }^{2}$ to distribute an incoming beam of light between the SiPM and a NIST ${ }^{3}$ certified calibrated photo-diode which is used to determine the absolute amount of light which reaches the SiPM. A similar method has been used in [3] to measure the PDE at single wavelengths; however, the setup used here allows PDE measurements over a wide spectral range, presently from 350 to $1000 \mathrm{~nm}$ (see section 2.2).

In order to guarantee that all light enters the $1 \mathrm{~mm}^{2}$ active area of the SiPM, the latter is placed behind an aperture with a diameter of $0.6 \mathrm{~mm}$. To centre the SiPM with respect to the aperture it is moved in the xy-plane until the signal is maximal.

While the SiPM is placed behind an aperture, the calibrated photo-diode used as a reference is directly mounted onto the sphere; the amount of light reaching the photo-diode is consequently much greater than that entering the SiPM. Therefore a light power ratio, $R_{0.6}$, is defined which needs to be determined. This was done using the calibrated photo-diode and measuring the light yield behind the aperture with respect to the case that it is directly mounted to the sphere. As expected, high values are observed for $R_{0.6}$ as shown in table 1 for different LEDs/laser diodes which deliver light at four different wavelengths.

\footnotetext{
${ }^{1}$ Calorimeter for the Linear Collider Experiment

${ }^{2}$ Newport Corporation, Model 819D-SL-3.3

${ }^{3}$ National Institute of Standards and Technology
} 
One additional benefit of a high power ratio is the partial compensation of the different sensitivities of the calibrated photo-diode and the SiPM, since the SiPM (Gain $=10^{5}-10^{6}$ ) is capable of detecting single photons, whereas the photo-diode $(\mathrm{Gain}=1)$ will only generate a measurable output for much higher light intensities.

For the PDE measurement, the laser diodes and LEDs are operated in a pulsed mode and the SiPM signal pulses are integrated with a charge to digital converter ${ }^{4}$ (QDC) which is triggered independently from the measured signal each time a light pulse is generated. For several values of the SiPM bias-voltage, charge spectra are recorded. These spectra serve as basis for the PDE determination described below. One example of such a spectrum is shown in figure 2 (left side); each peak corresponds to a certain number of fired pixels.

Before a PDE value is calculated, the breakdown-voltage, $U_{\text {break }}$, of the SiPM is determined. This is done by measuring the gain of the SiPM as a function of the bias-voltage (figure 2, right side); the gain is extracted using the distance between two consecutive photon-peaks in the charge spectra. By extrapolating the linear dependence between gain and bias-voltage to a gain of zero, $U_{\text {break }}$ is determined. The PDE results are then given as a function or for a particular value of the over-voltage $U_{\text {over }}=U_{\text {bias }}-U_{\text {break }}$.

In order to estimate the number of photons detected by the SiPM (photo-electrons), Poisson photo-statistics is used. This method has the advantage that it is insensitive to cross-talk and afterpulses and thus allows to determine an absolute value for the PDE. It is based on measuring the probability $P\left(0, n_{p e}\right)$ to detect zero photons, where $n_{p e}$ is the mean number of photo-electrons seen.

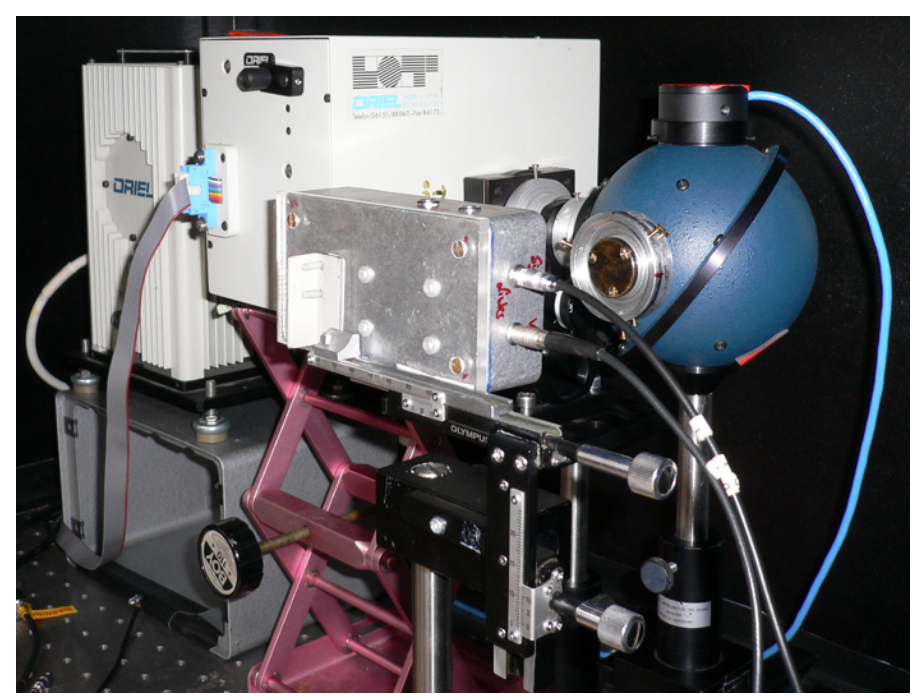

Figure 1: Experimental setup for the PDE measurement consisting of the a xenon lamp, a monochromator and an integrating sphere. On the top port of the sphere a NIST certified calibrated photo-diode is attached. The aluminium box in front contains the SiPM under test and can be moved in the xy-plane to align the SiPM. For the statistical analysis the xenon lamp and the monochromator can be replaced by a pulsed light source.

\footnotetext{
${ }^{4}$ LeCroy Model 2249A
} 
Using the standard Poisson probability distribution yields:

$$
\begin{aligned}
P\left(0, n_{p e}\right) & =e^{-n_{p e}} \\
\rightarrow n_{p e} & =-\ln \left(P\left(0, n_{p e}\right)\right) \\
& =-\ln \left(\frac{N_{p e d}}{N_{\text {tot }}}\right)+\ln \left(\frac{N_{p e d}^{\text {dark }}}{N_{\text {tot }}^{\text {dark }}}\right)
\end{aligned}
$$

here, $N_{\text {tot }}$ and $N_{\text {ped }}$ refer to the total number of events and the number of times zero photons have been detected, respectively. $N_{\text {ped }}$ is determined by fitting a Gaussian to the pedestal peak (figure 2 , left side, shaded region) to determine a $\pm 3 \sigma$ range for the pedestal peak; the number of entries within this range represents the number of "zero-photon" events, $N_{p e d}$. For $N_{t o t}$ the full spectrum is integrated.

The second term in equation 2.1 accounts for the number of detected photo-electrons due to the thermal rate. $N_{\text {tot }}^{\text {dark }}$ and $N_{\text {ped }}^{\text {dark }}$ can be estimated form the corresponding charge spectrum when the light source is switched off and the QDC is triggered randomly.

The PDE is then calculated from $n_{p e}$ and considering the power ratio $R_{0.6}$, the period of the light-pulses, $T=30 \mu$ s and the optical power, $P_{o p t}$, measured with the calibrated photo-diode:

$$
P D E=\frac{n_{p e} \cdot R_{0.6} / T}{P_{o p t} /\left(h \cdot \frac{c}{\lambda}\right)}
$$

where $h$ is the Planck constant, $\lambda$ is the wavelength and $c$ is the speed of light. As an example figure 3 (left side) shows the measured PDE as a function of the over-voltage for an MPPC ${ }^{5}$ with 1600 pixels. As expected the PDE rises with increasing over-voltage, and a clear saturation behaviour of the PDE is seen at high values of $U_{\text {over }}$, also observed in [4].

\subsection{Relative Sensitivity Measurement}

As the statistical analysis requires a pulsed light source it is done only for a limited number of wavelength values shown in table 1. Thus, in order to measure and characterise the PDE over a wide spectral range a second method is used which allows to determine the relative spectral sensitivity in the range from $\lambda=350$ to $1000 \mathrm{~nm}$. The experimental setup is similar to the one described in section 2.1 with the difference that instead of laser diodes and LEDs, a xenon lamp in combination with a monochromator is used to generate continuous-wave monochromatic light; the SiPM current is measured using a pico-ampere meter. With this method, optical cross-talk and

\begin{tabular}{ccc} 
Type & Centre wavelength $[\mathrm{nm}]$ & Ratio $R_{0.6}$ \\
\hline LED & 465 & $4200 \pm 20$ \\
Laser diode & 633 & $3852 \pm 18$ \\
Laser diode & 775 & $4328 \pm 7$ \\
LED & 870 & $4625 \pm 55$ \\
\hline
\end{tabular}

Table 1: Measured power ratios for an aperture of $\varnothing 0.6 \mathrm{~mm}$.

\footnotetext{
${ }^{5}$ Multi Pixel Photon Counter, HAMAMATSU photonics company
} 

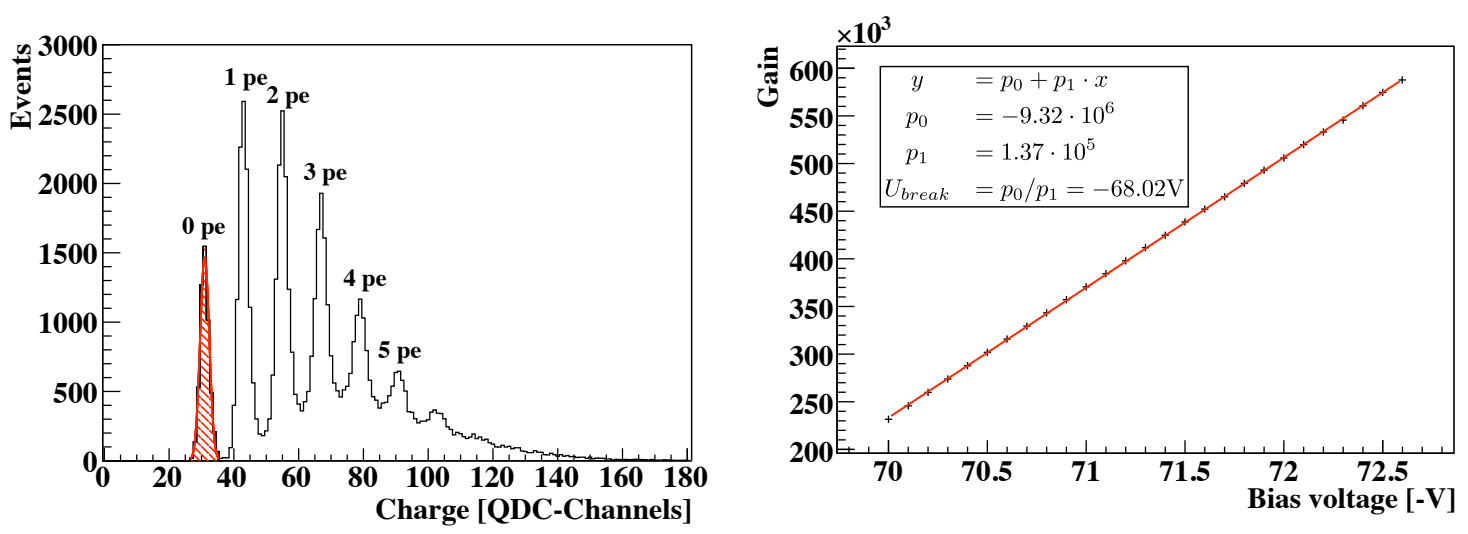

Figure 2: (left) Single photo-electron spectrum recorded with an MPPC with 1600 Pixels. Each peak corresponds to a certain number of photo-electrons (pe). (right) Measured gain as a function of the biasvoltage for an MPPC with 1600 pixels (S10362-11-025C). The breakdown-voltage was calculated form the fit parameters.

after-pulses cannot be separated from the actual signal. Hence the measured values of the spectral sensitivity are higher than the actual PDE. Nevertheless, by scaling the measured curves to the absolute PDE values, determined with the statistical analysis, it is possible to acquire an absolute PDE over the full spectral range from 350 to $1000 \mathrm{~nm}$. The final result of our PDE measurement is shown in figure 3 (right side) for three different types of SiPMs at the maximum over-voltage for which the statistical analysis can be applied; this is individually determined for each of the sensors (see figure); the filled markers indicate the result of the statistical analysis whereas the open ones show the relative measurement. Note that the re-scaling of the relative measurement has been done with respect to the result at $633 \mathrm{~nm}$ from the statistical analysis. The fact that the corresponding measurements at 465,775 and $870 \mathrm{~nm}$ also fall on the re-scaled curve can be considered as an additional cross check proving the reliability of the measurements.

The results show that both MPPCs from HAMAMATSU are peaked in the blue region around $450 \mathrm{~nm}$. However, the 400 pixel MPPC has a higher PDE than the 1600 pixel device due to the large geometrical fill factor $\varepsilon_{g e o}$. In general the PDE of a SiPM can be factorised as follows:

$$
P D E=\varepsilon_{\text {geo }} \cdot Q E \cdot \varepsilon_{\text {trigger }}
$$

where $Q E$ is the quantum efficiency, i.e. the probability that an electron hole pair is generated, and $\varepsilon_{\text {trigger }}$ is the summed probability of the pair to trigger electrical break-down (Geiger break-down).

For the SensL device (SPM) the peak value of the PDE value is shifted into the green region. This is due to the different doping structures of MPPC ( $p$ on $n$ type) and SPM ( $n$ on p type) as e.g. described in detail in $[5,6]$.

\section{Uniformity Scans}

To study the SiPM uniformity, i.e. the spacial variations in sensitivity, gain and the cross-talk probability, two additional experimental setups have been used, one located at the Kirchhoff-Institut 

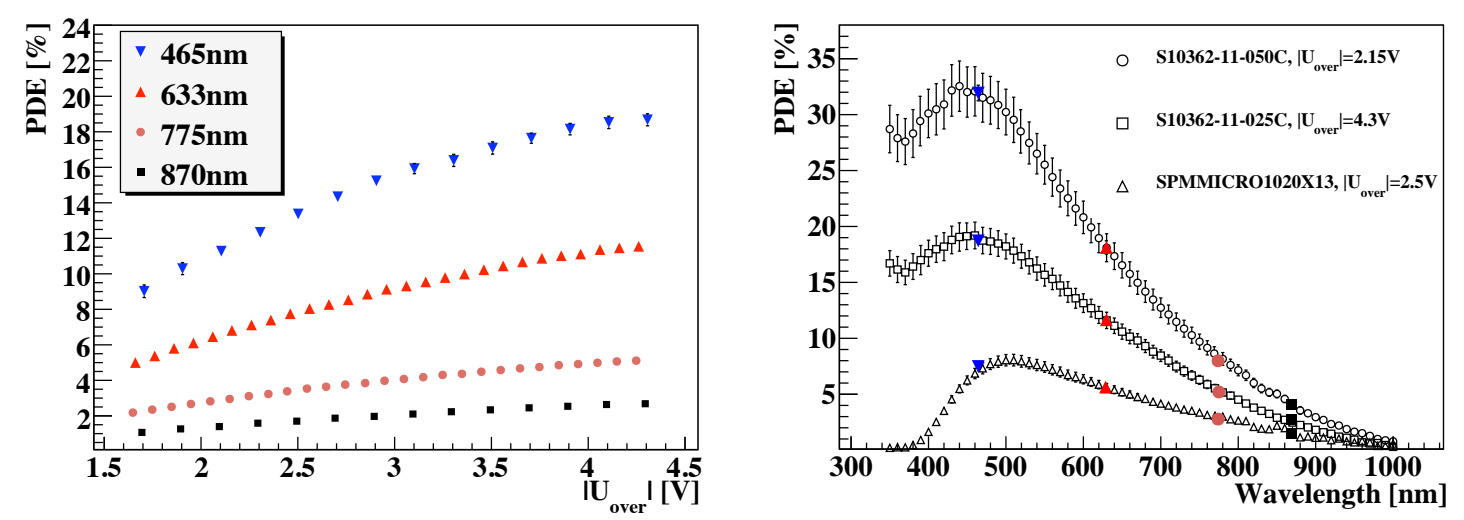

Figure 3: (left) PDE of the HAMAMATSU S10362-11-025C as a function of the over-voltage at four different wavelength of light. (right) PDE of the HAMAMATSU S10362-11-025C (25 $\mu \mathrm{m}$ pitch), S1036211-050C (50 $\mu \mathrm{m}$ pitch) and SensL SPMMICRO1020X13 (20 $\mu \mathrm{m}$ pitch) as a function of the wavelength of light at room temperature $\left(25 \pm 1.5^{\circ} \mathrm{C}\right)$. The filled markers indicate the PDE values determined in section 2.1 (see left plot for colour code). The curves have been scaled to the maximum PDE value at $633 \mathrm{~nm}$.

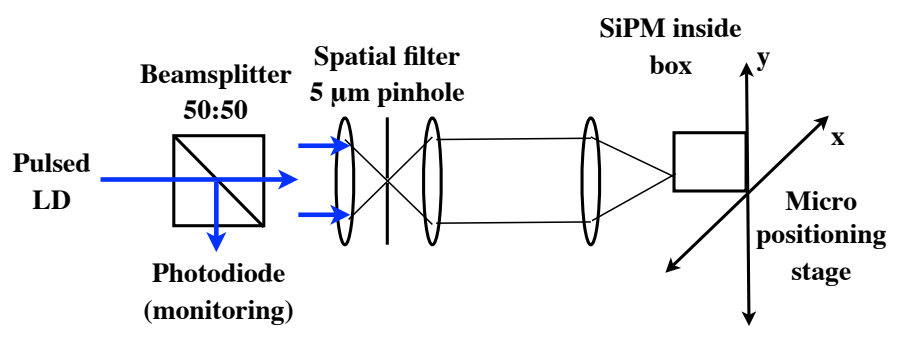

Figure 4: Schematic view of the setup using a focussed light spot to raster scan the active area of the SiPM.

für Physik in Heidelberg and the other at the Max-Planck Institute in Munich. The first setup is schematically shown in figure 4 and uses a focussed light spot to raster scan the SiPMs. A pulsed laser diode generates short $(\sim 2 \mathrm{~ns})$ light pulses divided by a beam splitter into two separate beams, one of which is monitored by a photo-diode for long term stability while the other passes a spatial filter and is focussed onto the active area of the SiPM. The SiPM is installed inside a protective box that is mounted on a xy-positioning stage which allows to position it with micrometer accuracy relative to the beam used for scanning the device. At each geometrical position a charge histogram with 10.000 entries is recorded and evaluated with the same method described in section 2.1. In this way the position dependent sensitivity (corrected for the influences of optical cross-talk and after-pulses) can be measured. Furthermore, the acquired charge histograms allow to calculate the gain (distance between the peaks) as well as the cross-talk probability where the latter is defined here as the ratio of the number of events with a measured charge above the single-photo peak (compare figure 2, left side) and the number of total signal events above the pedestal (non-zero photon events); for a device with no cross-talk only single-photon events are expected, if only one pixel is illuminated. For the measurement a short QCD-gate is set $(\sim 30 \mathrm{~ns})$ in order to reduce the impact of to the fast component of after-pulses which has been observed in [7].

The results of the uniformity scans for the MPPC (S10362-11-100C and S10362-11-050C) 
are depicted in figures 5 - 7. Some variations of the sensitivity (fig. 5) and the gain (fig. 6) are observed. However, temperature differences may have to be taken into account due to the relative long measurement time $(\sim 40 \mathrm{~h})$, and the uniformity might be better then indicated by the plots. The optical cross-talk probability (fig. 7) is higher in the centre of the device as expected due to the increased number of neighbouring pixels. A variation of the cross-talk probability within single pixels is also observed with lower values at the edges of pixels facing outwards and higher values at inside boarders.

Independent uniformity measurements have been done at the Max-Planck Institute for Physics in Munich using a similar setup. For these measurements a low intensity continuous-wave laser was used for device scanning, and the pulse rate was determined using a threshold counter. The results for a HAMAMATSU S10362-11-100C are shown in figure 8 where the upper left plot shows the count rate of signals with an amplitude above a threshold of 0.5 photo-electrons (pe). Note that the irregular structure in $\mathrm{x}$-direction is a feature of the scanning process and does not reflect the geometrical layout of the MPPC. The observed count rate is higher at the centre of the active area, i.e. in the region where higher cross-talk is expected and also observed by the previous measurement. The upper right plot shows the count rate above a threshold of 0.5 pe minus the rate of events with an amplitude higher then $1.5 \mathrm{pe}$; it thus reflects the rate for events where only one pixel has fired. In this case an improved uniformity is observed indicating that the higher count rate in the upper left plot of figure 8 is indeed due to optical cross-talk. Finally, the lower plot of figure 8 shows the cross-talk probability defined now as the ratio of the count rates above 1.5 and 0.5 pe. Qualitatively, the result is the same as the one shown in figure 7, although quantitative differences, which may occur since different MPPC samples have been tested and due to the different methods used, still have to be understood.

\section{Conclusion}

Several experimental results characterising different aspects of Silicon Photomultipliers (SiPM) have been presented; emphasise has been put on the measurement methods used to determine the Photon-Detection-Efficiency (PDE) of such sensors as well as device uniformity studies. The results have been obtained in the framework of the analogue hadron calorimeter project of the CALICE collaboration which uses SiPMs for reading out the scintillator tiles of their highly granular scintillator-based calorimeters. A precise knowledge of SiPM properties is crucial for a decision on which device is best suited for such an application. Potential candidates have and will thus be characterised using the experimental setups. Here results from three sample devices, two MPPCs from HAMAMATSU ${ }^{6}$ and one device from SensL ${ }^{7}$ are presented and compared.

The PDE measurements are done over a wide spectral range from 350 to $1000 \mathrm{~nm}$. For the HAMAMATSU MPPCs the results show a maximum PDE in the blue region at about $450 \mathrm{~nm}$; as expected from the different geometrical fill factors the PDE is larger for the 400 pixel device than for the one with 1600 pixels, the latter, however, providing a large dynamical range. The maximum PDE for the SPM from SensL is shifted into the green and measured to be at about $500 \mathrm{~nm}$. The

\footnotetext{
${ }^{6}$ S10362-11-100C, S10362-11-050C, S10362-11-025C

${ }^{7}$ SPMMICRO1020X13
} 

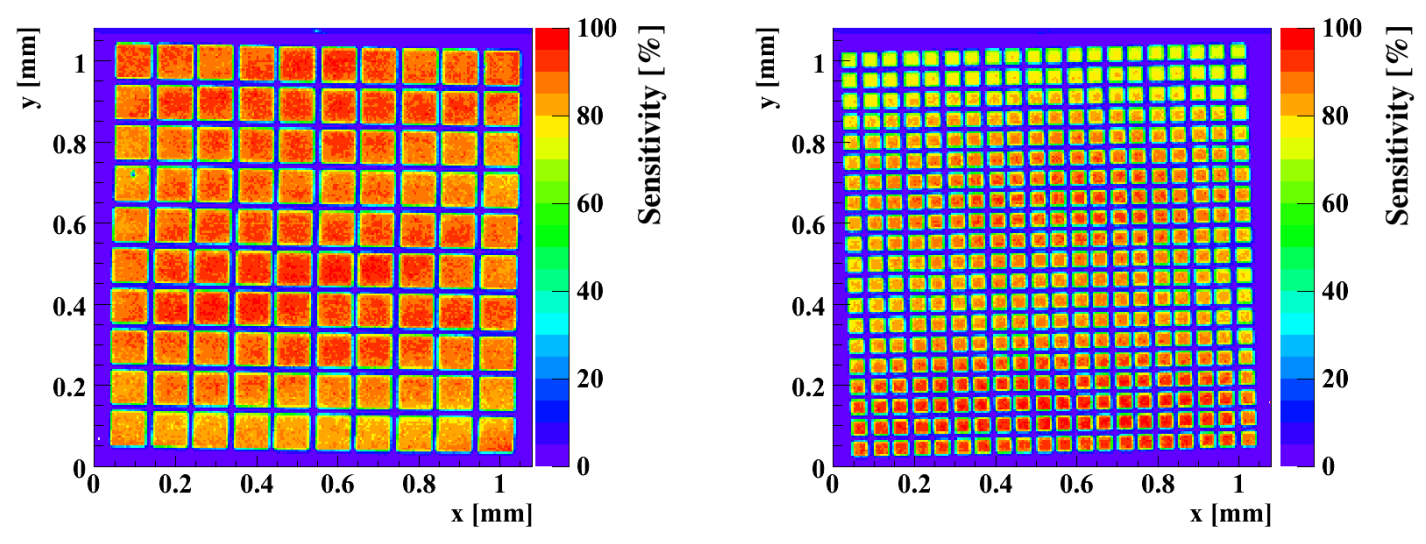

Figure 5: (left) Relative sensitivity map of an HAMAMATSU S10362-100C (100 $\mu \mathrm{m}$ pitch). (right) Relative sensitivity map of an HAMAMATSU S10362-050C (50 $\mu \mathrm{m}$ pitch). Device operated at nominal voltage.
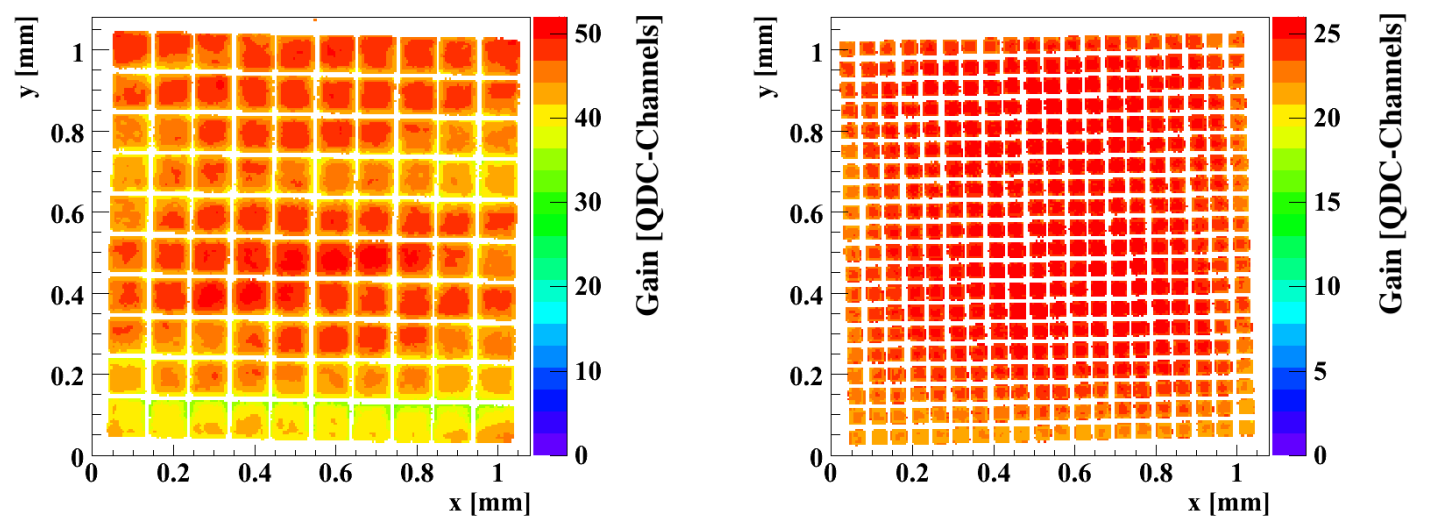

Figure 6: (left) Gain map of an HAMAMATSU S10362-100C (100 $\mu \mathrm{m}$ pitch). (right) Gain map of an HAMAMATSU S10362-050C (50 $\mu \mathrm{m}$ pitch). Parts with a sensitivity smaller than $50 \%$ were set to zero.
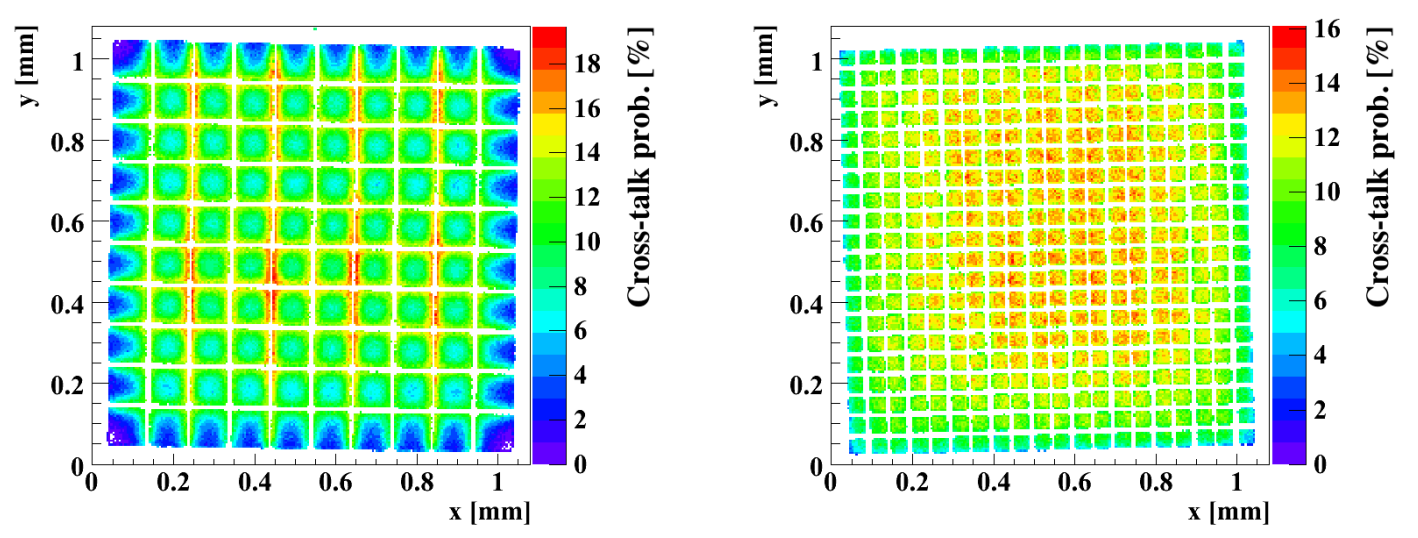

Figure 7: (left) Cross-talk map of an HAMAMATSU S10362-100C (100 $\mu \mathrm{m}$ pitch). (right) Cross-talk map of an HAMAMATSU S10362-050C (50 $\mu \mathrm{m}$ pitch). Parts with a sensitivity smaller than $50 \%$ were set to zero. 

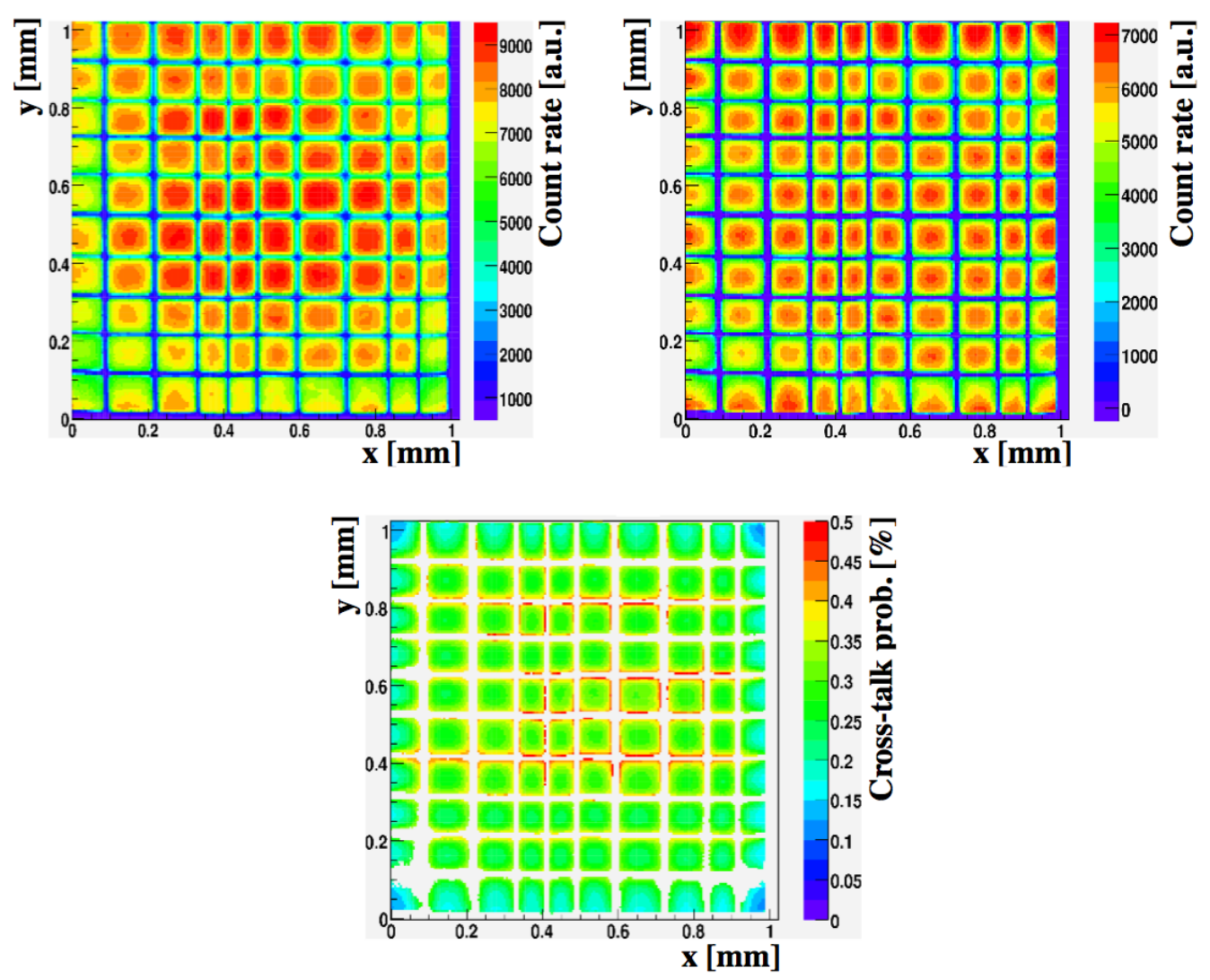

Figure 8: (upper left) Count rate as a function of the geometrical position for an MPPC (S10362-11-100C). (upper right) Count rate for 0.5 pe threshold minus count rate for 1.5 pe threshold; see text for details. (bottom) Cross-talk map derived from the ratio of of the count rates above 1.5 and 0.5 pe (see text). A cut-off has been applied for high cross-talk values observed in between pixels.

wavelengths at which the peak sensitivities are observed agree with the values officially published by the two companies.

Concerning the uniformity measurements, results from two different experimental setups have been reported. They show that the tested sensors from HAMAMATSU are fairly uniform concerning sensitivity and gain. For the cross-talk probability higher values are observed in the centre of the devices where pixels have a higher number of neighbours. An observed inter-pixel variation of the cross-talk can be explained by a higher (lower) probability for producing an extra avalanche in a neighbouring pixel if it is closer (further) away from the probed location.

\section{References}

[1] V. Andreev et al., A high-granularity plastic scintillator tile hadronic calorimeter with APD readout for a linear collider detector, Nucl. Instr. and Meth. A 564 144-154 (2006).

[2] International linear collider reference design report, ILC-REPORT-2007-001 (2007).

[3] A. N. Otte et al., A Measurement of the Photon Detection Efficiency of Silicon Photomultipliers, Nucl. Instr. and Meth. A 567 360-363 (2006). 
[4] S. Uozumi et al., Development and study of the multi pixel photon counter, Nucl. Instr. and Meth. A 581 427-432 (2007).

[5] D. Renker, E. Lorenz, Advances in solid state photon detectors, JINST 4 P04004 (2009).

[6] William G. Oldham et al., Triggering Phenomena in Avalanche Diodes, IEEE Trans. Electron Dev. 19 1056-1060 (1972).

[7] Y. Du, F. Retière, After-pulsing and cross-talk in multi-pixel photon counters, Nucl. Instr. and Meth. A 596 396-401 (2008). 